\title{
Bericht über die Podiumsdiskussion „Die Aufklärung von Straftaten gegen das Völkerrecht als Voraussetzung für den Aufbau einer effizienten Justiz" in Stuttgart am 9. November 2017
}

\author{
Aniello Ambrosio*
}

Im Oktober 2014 besuchten sieben Rechtsanwälte, Richter und Staatsanwälte aus Burundi, der Demokratischen Republik Kongo und Ruanda Stuttgart und erhielten von örtlichen Justizjuristen einen Einblick in den baden-württembergischen Justizalltag. ${ }^{1}$ Im Februar 2016 konnten sodann sieben Richter und Staatsanwälte aus Stuttgart und Koblenz zu einem Gegenbesuch in die Demokratische Republik Kongo und nach Ruanda reisen. ${ }^{2}$ In diesem Kontext hielten die deutschen Tandempartner auch einen Vortrag an der Juristischen Fakultät der Universität Kigali (Ruanda). Die anschließende Diskussion mit Vertretern der Fakultät und Jurastudenten drehte sich vor dem Hintergrund zahlreicher Verfahren nach dem Völkerstrafgesetzbuch (VStGB) vor den Oberlandesgerichten Stuttgart, ${ }^{3}$ Frankfurt am Main ${ }^{4}$ und Düsseldorf ${ }^{5}$ um Fragen der Auslieferung beschuldigter ruandischer Staatsangehöriger von deutschen Justizbehörden an die ruandische Generalstaatsanwaltschaft. Von Seiten der Studenten wurde vielfach Unverständnis für die zurückhaltende Auslieferungspraxis deutscher Behörden und die Zweckmäßigkeit von Strafverfahren in der Bundesrepublik wegen in der Demokratischen Republik Kongo oder in Ruanda begangener Straftaten laut. Dass einzelne Angeklagte die deutsche Staatsangehörigkeit annahmen, wurde von zahlreichen ruandischen Studenten als bewusster Schutz der Angeklagten vor Strafverfolgung in Ruanda aufgefasst.

Den deutschen Tandempartnern der 1. Generation war es daher ein Anliegen, den Austausch zu vertiefen und mit dem sehr aktuellen Thema der Aufklärung von Straftaten gegen

* Staatsanwalt Aniello Ambrosio ist bei der Staatsanwaltschaft Stuttgart beschäftigt und zurzeit als Parlamentarischer Berater an den Landtag von Baden-Württemberg abgeordnet. Er war Teilnehmer des Deutsch-Afrikanischen Juristenaustausches in den Jahren 2014 bis 2016.

1 Kahombo, Balingene, Summary Report on the 'Exchange Programme between Young Lawyers from Burundi, Rwanda and the Democratic Republic of Congo and the Judiciary of Baden-Württemberg (Germany)' from 5 to 18 October 2014, in: Recht in Afrika - Law in Africa - Droit en Afrique 17 (2014), S. 228-235.

2 Vgl. Ambrosio, Aniello et al., Report on the Exchange Programme between Young Lawyers from Central and East Africa and the Judiciary of Baden-Württemberg and Rheinland-Pfalz, Germany, in Lubumbashi (Democratic Republic of Congo), Kigali (Rwanda) and Nairobi (Kenya) from 6 to 15 February 2016, in: Recht in Afrika - Law in Africa - Droit en Afrique 19 (2016), S. 94-101.

3 Urt. v. 28.9.2015, 5 - 3 StE 6/10 - juris.

4 Urt. v. 29.12.2015, $4-3 \mathrm{StE} 4 / 10-4-1 / 15$ - juris.

5 Urt. v. 5.12.2014, III - 6a StS 1/13 - unveröffentlicht. 
das Völkerrecht zu verknüpfen. Daraus entstand die Idee, mit einer Podiumsdiskussion mit renommierten Gästen zum Thema „Die Aufklärung von Straftaten gegen das Völkerrecht als Voraussetzung für den Aufbau einer effizienten Justiz“ auf den deutsch-afrikanischen Juristenaustausch aufmerksam zu machen. Unterstützt wurden die Juristen dabei vom Dynamischen Europapool Baden-Württemberg, von der Gesellschaft für Afrikanisches Recht e.V., von der Stiftung Entwicklungs-Zusammenarbeit (SEZ) Baden-Württemberg, von der Robert-Bosch-Stiftung, vom Staatsministerium und vom Ministerium der Justiz und für Europa Baden-Württemberg, von der BW-Bank, von Engagement Global und vom Bundesministerium für wirtschaftliche Zusammenarbeit (BMZ).

Dank der Organisation von Prof. Dr. Hartmut Hamann, Vorstandsmitglied der Gesellschaft für Afrikanisches Recht, und der Unterstützung durch die Kooperationspartner, v.a. die Robert-Bosch-Stiftung, war schnell klar, dass im Herbst 2017 erneut Juristen aus dem Partnerland von Baden-Württemberg, Burundi und dessen Nachbarstaaten nach Stuttgart reisen werden. Vier Richter und Staatsanwälte aus Burundi, der Demokratischen Republik Kongo, Ruanda und Tansania nahmen vom 5. bis 12. November 2017 an einem abwechslungsreichen Programm in Stuttgart sowie an der Jahrestagung der Gesellschaft für Afrikanisches Recht in Freiburg zum Thema „Armed Conflict in Africa: Legal and Political Measures for Protecting Displaced People and Preventing Forced Migration“ teil. Ein Programmpunkt war dabei die Teilnahme der neuen deutsch-afrikanischen Tandempartner an der vorgenannten Podiumsdiskussion.

Philipp Keil, Geschäftsführender Vorstand der SEZ, hieß die mit etwa 100 Gästen gut besuchte Veranstaltung in den Räumlichkeiten der BW-Bank in Stuttgart willkommen. Prof. Dr. Hartmut Hamann führte als Initiator des deutsch-afrikanischen Juristenaustausches kurz in dessen Entstehungsgeschichte und Ziele ein. ${ }^{6}$ Sodann eröffnete Ministerialdirektor Elmar Steinbacher als Vertreter des Ministeriums der Justiz und für Europa des Landes BadenWürttemberg die Diskussionsrunde.

Seit 15 Jahren existiert der Internationale Strafgerichtshof (IStGH) in Den Haag - ,ein entscheidender Beitrag zu einer Welt ohne Straflosigkeit“". ${ }^{7}$ Aber auch durch vom UN-Sicherheitsrat geschaffene Ad-hoc-Strafgerichtshöfe, wie für das ehemalige Jugoslawien oder Ruanda, sowie durch nationale Gerichte, wie vor dem Oberlandesgericht Stuttgart im „Ruanda-Prozess“, wird das Völkerstrafrecht durchgesetzt. Angesichts beschränkter Mittel der Ermittlungsbehörden und Gerichte, langwieriger Verfahren oder nachlassender politischer Unterstützung wird die Wirkungskraft des Völkerstrafrechts aber auch in Zweifel gezogen.

6 Vgl. hierzu Sippel, Harald, Förderung der Justiz in Afrika: Austauschprogramm zwischen BadenWürttemberg und zentralafrikanischen Staaten, in: Recht in Afrika - Law in Africa - Droit en Afrique 17 (2014), S. 113-115; Sippel, Harald, Förderung der Justiz in Afrika: Konferenz zur Unabhängigkeit und Effizienz der Justiz in Lubumbashi und Fortsetzung des juristischen Austauschprogramms zwischen Baden-Württemberg und zentralafrikanischen Staaten, in: Recht in Afrika - Law in Africa - Droit en Afrique 18 (2015), S. 139-142.

7 Vgl. Kofi Annan, Rede vor dem Deutschen Bundestag am 28.2.2002, http://www.unric.org/de/press emitteilungen/4000 [zuletzt abgerufen am 19.12.2017]. 
Mit Experten aus der DR Kongo, der Schweiz sowie aus Deutschland wurde u. a. der Frage nachgegangen, inwieweit die Durchsetzung des Völkerstrafrechts das Vertrauen der Bevölkerung in die Justiz stärken kann und ob die Instrumente des Völkerstrafrechts als Vorbild für ein nationales Justizsystem dienen können.

Für die Debatte konnten gewonnen werden: Prof. Dr. Kai Ambos, Richter am KosovoSondertribunal, Leiter der Abteilung für ausländisches und internationales Strafrecht und Professor für Strafrecht, Strafprozessrecht, Rechtsvergleichung und internationales Strafrecht am Institut für Kriminalwissenschaften der Georg-August-Universität Göttingen, Jürgen Hettich, Vorsitzender Richter am Oberlandesgericht Stuttgart, Balingene Kahombo, vormals Staatsanwalt am Parquet près le Tribunal d'Instance in Kinshasa/DR Kongo, derzeit im Rahmen eines Promotionsstudiums an der Freien Universität Berlin, und Stefan Waespi, von 1996 bis 2010 Ankläger beim Internationalen Strafgericht für das ehemalige Jugoslawien, Staatsanwalt des Bundes bei der Schweizerischen Bundesstaatsanwaltschaft in Bern, zuständig für Rechtshilfe und Völkerstrafrecht. Die Moderation übernahm die Leiterin der Justizpressekonferenz, der SWR-Rechtsredaktion sowie der ARD-Rechtsredaktion Hörfunk, Gigi Deppe.

Frau Deppe nahm den sog. „Ruanda-Prozess“ vor dem OLG Stuttgart zum Ausgangspunkt für Fragen an Richter am Oberlandesgericht Hettich. Dieser äußerte sich zunächst kritisch zur Zweckmäßigkeit von Strafverfahren wegen Verbrechen nach dem Völkerstrafgesetzbuch (VStGB). Solche Verfahren seien mit den Strengbeweismitteln und den Ordnungsvorschriften der StPO nicht durchführbar. So seien Zeugenaussagen wegen der kriegsartigen Zustände in der Demokratischen Republik Kongo, Repression und Druck nicht verwertbar gewesen. Lösungen für diese Herausforderung könnten aus der Übernahme der Prozessordnung des IStGH in deutsches Recht oder aus der Änderung der StPO für solche Verfahren resultieren. Zudem forderte er eindringlich eine bessere Zusammenarbeit von IStGH und Generalbundesanwaltschaft (GBA). Vielfach würden Zeugen von den nationalen und internationalen Strafverfolgungsbehörden mehrfach vernommen. Dies habe bewusste oder unbewusste Erinnerungslücken, weitergehende Traumatisierung und Unwillen von Opferzeugen an der Sachverhaltsaufklärung zur Folge. Jedenfalls teilweise wurden auch Rechtshilfeersuchen durch den IStGH nicht beantwortet.

Dieser Einschätzung widersprach Professor Ambos, der die derzeitige internationale justizielle Zusammenarbeit für funktional erachtet. Staatsanwalt des Bundes Waespi stellte dar, dass die Schweizerische Bundesanwaltschaft engagiert Straftaten gegen das Völkerrecht verfolge, hierfür aber nur auf begrenzte Personalressourcen zurückgreifen könne. Aktuell werde in Bern wegen in Gambia begangener Straftaten ermittelt. Dabei würden Zeugen eingeflogen und vom Staatsanwalt unter Anwesenheit der weiteren Beteiligten vernommen. Entscheidend für die Akzeptanz des Verfahrens und der gerichtlichen Entscheidung sei gerade die Teilnahme aller Beteiligter an den Vernehmungen und die Herstellung einer für die Opferzeugen angenehmen Verhandlungsatmosphäre. Ein Wermutstropfen sei jedoch der Umstand, dass die Schweizer Richter wahrscheinlich nur aufgrund der Aktenlage entschieden. Herr Waespi erachtete es als schwierig, in Europa Strafverfahren zur Aufklärung 
von Straftaten in Afrika zu führen, da nur anwesenden Angeklagten der Prozess gemacht werden könne. Diese müssten sich folglich zufällig in Europa aufhalten und dann auch noch als mögliche Täter erkannt werden.

Balingene Kahombo brachte die kongolesische Sicht der Dinge in die Diskussion ein: In Deutschland seien bisher nur „kleine“, eher unbedeutende Fälle entschieden worden. Die Urteile hätten nur geringe Auswirkungen auf die (kriminal-)politische und soziale Situation vor Ort. Es sei aber auch unschädlich, dass entsprechende Strafverfahren in Europa geführt werden. In der Demokratischen Republik Kongo fehle es zur Aburteilung von Straftaten gegen das Völkerstrafrecht v.a. an der Infrastruktur und am Personal. Die Europäische Union müsse es sich daher zum Ziel machen, die Rechtsstaatlichkeit in den afrikanischen Staaten zu stärken.

Professor Ambos entgegnete, dass es nicht sein könne, dass Europa insoweit die Strafverfolgung übernehme. Ziel müsse es vielmehr sein, dass die Europäer den afrikanischen Staaten dabei helfen, die Infrastruktur vor Ort aufzubauen und nur unterstützend zu wirken. Recht und Rechtsprechung seien stets durch die jeweilige Amtssprache und die örtlichen Gebräuche geprägt, die ein Europäer nicht kenne.

Alle Referenten forderten von der Justizverwaltung, dass Urteile oder jedenfalls die zugehörigen Pressemitteilungen in Verfahren nach dem VStGB in die englische Sprache übersetzt werden müssten, wenn Gerichte in Europa schon Straftaten gegen das Völkerrecht in anderen Teilen der Erde aburteilten.

In der Schlussrunde betonte Professor Ambos die Bedeutung des wechselseitigen Austauschs der verschiedenen Justizsysteme für das globale Miteinander. Die Justizverwaltungen könnten voneinander lernen. So habe Brasilien bereits vor zehn Jahren die elektronische Akte eingeführt. Auch sei es sinnvoll, Tandems zwischen deutschen und afrikanischen Justizjuristen zu bilden und diese den Berufsalltag in den beteiligten Staaten gemeinsam durchlaufen zu lassen. Richter am Oberlandesgericht Hettich forderte, die Strafverfahren in der Bundesrepublik handhabbarer zu machen. Zum Abschluss zeigte Staatsanwalt des Bundes Waespi eindrucksvolle Bilder aus seiner Tätigkeit in der Demokratischen Republik Kongo in einem vom UN-Sicherheitsrat eingerichteten Projekt. Im Rahmen des UN-Einsatzes MONUSCO beriet er die lokale Justiz im Zuge der Ermittlung von Verbrechen des Völkerstrafrechts. Im Zentrum stand die Organisation von sog. „mobile courts“, das heißt ad hoc-Gerichten an weitabgelegenen Orten, die bei der lokalen Bevölkerung große Aufmerksamkeit fanden. Die Fotopräsentation mit zugehörigem Erfahrungsbericht rundete die Diskussion mit einem sehr anschaulichen Beispiel für die internationale Zusammenarbeit auf dem Gebiet der Justiz ab. 